\title{
Société Française d'Allergie Séance du 17 novembre 1959
}

Méthode de désensibilisation par doses subinti·antes chez un sujet sensibilisé à la pécicilline

MM. Pasteur Vallery-Radot, Domart, Wolfromm et Hazard rapportent un cas de maladie d'Osler où le malade, allergique à la pénicilline, - comme le démon-traient les accidents antérieurs à la suite d'injections de pénicilline - devait être rapide-ment désensibilisé à cet antibiotique, le seul efficace dans l'affection qu'il présentait.

Ces auteurs ont commence par injecter 20 unites, toutes les 2 heures, par voie intradermique, puis des doses de plus en plus fortes, par la voie hypodermique, jusqu'au 6ème jour (6000 U. en 24 heures) et ensuite par la voie intramusculaire: le 7ème jour, $1000 \mathrm{U}$, toutes les 3 heures, et, à partir du 8ème jour, deux fois par jour, des doses pro-gressivement croissantes pour atteindre la dose plafond quotidienne de 30 millions d'unités, renouvelée chaque jour à partir du 17ème jour, et totaliser 933 millions d'unités, le 42ème jour de traitement.

Cette désensibilisation fut faite sous couvert d'un antihistaminique pendant tout le traitement. Reaction anaphylactique générale severe à la suite de Гapplication d'un suppositoire à la pénicilline

M. Halpern signale le cas d'une jeune fille asthmatique chez laquelle Гintroduc-tion d'un suppositoire contenant 100000 unites de pénicilline a provoqué un veritable choc anaphylactique avec collapsus cardio-vasculaire, perte de connaissance, ayant nécessité une medication héroïque sous forme d'injection intraveineuse de Néosyné-phrine et d'injection intramusculaire de Phénergan. Avec ce traitement, la situation à été rapidement rétablie. L'auteur insiste sur la nécessité de dépister, par Гinterrogatoire, les indications pouvant faire soupçonner une sensibilisation antérieure à la pénicilline; cette malade avait eu en effet quelques accidents allergiques à la suite d'aérosols de pénicilline, 7 ans auparavant. II insiste aussi sur Faction rapide de la Néosynéphrine dans les cas de collapsus cardio-vasculaire.

Allergie à Taspirine; hémorragies digestives puis asthme ou urticaire ou oedème de Quincke M. Pierre Blamoutier rapporte sept observations de sujets allergiques à $\Gamma$ aspirine, qui présentaient, après prise de ce medicament, d'abord des troubles digestifs divers traduisant Tirritation souvent intense produite par le contact de Tacide acétyl salicylique avec la muqueuse de Гestomac, des hémorragies gastriques plus ou moins importantes en particulier, puis moins de 24 heures après, une crise d'asthme, de prurit generalise, d'urticaire ou d'ædème de Quincke. Ces manifestations ne se produisaient qu'après Tabsorption d'aspirine et, chaque fois, Ton trouvait une reaction à la benzi-drine positive dans les selles.

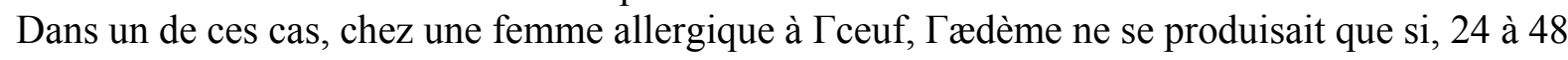
heures avant, elle avait pris de Гaspirine. Sans Гirritation provoquée par celle-ci sur la muqueuse gastrique, Гceuf était bien toléré, aucun cedème n'apparais-sait. 234

Proceedings - Gesellschaftsberichte - Sociétés

Etude d'un anticorps allergique actif en presence de para-amino-salicylate de soude (P.A.S.) contre les hématies, Ies leucocytes et lee 
plaquettes humaines

Mme Bergerot-Blondel, MM. Denecheau, Schicklin et Dausset ont mis en evidence, dans le serum d'un malade traité par le P.A.S. sodique, ayant présenté des chocs de plus en plus graves à la suite de plusieurs series de perfusions intra-veineuses, une substance capable d'agglutiner les hématies compatibles au taux de 1/128, et, à un titre moindre, les leucocytes et les plaquettes. Cette substance inhibe la retraction du caillot. Elle se trouve exclusivement dans la fraction $\gamma$ globulinique du serum. L'étude des radicaux chimiques permet de conclure à la presence obligatoire d'un noyau benzénique et d'un Coon a en position I d'une part, d'autre part à un deuxième radical $\mathrm{OH}$ ou $\mathrm{OCH} 3$ en position 2, ou d'un $\mathrm{NH} 2$ en position 4. La reaction ne peut se produire que si trois elements sont rassemblés: serum du malade, element figure et P.A.S. II s'établit ainsi deux liaisons: P.A.S.-element figure et P.A.S.-anticorps.

II semble bien s'agir d'un anticorps allergique réclamant la presence d'un aller-gène lie à une protéine pour donner un antigène. Cet anticorps responsable de chocs graves, différencie totalement ce cas des anémies hémolytiques brutales, parfois mortelles, apparaissant dès Гadministration du P.A.S., sous différentes formes, et qui relèvent d'un autre mécanisme. Sensibilisation des organes à muscles lisses «in vitro»

MM. Halpern, Binaghi, Liacopoulos et Mme Liacopoulos-Briot rapportent le résultat obtenu avec une nouvelle technique qui permet de fixer les anticorps sensibili-sants sur des organes isolés, en survie «in vitro». La reaction de l'organe ainsi sensibilisé «in vitro» à $\Gamma$ addition de Гantigène est spécifique. Le degré de sensibilisation est fonc-tion, d'une part, de la durée de contact, et, d'autre part, de la concentration en anticorps. Les anticorps, une fois fixes, ne peuvent pas être detaches par un simple lavage, même prolongé, avec des solutions physiologiques. Cependant, on peut obtenir un déplacement des anticorps par competition avec des gamma-globulines non spécifiques. L'acide carbonique empêche la fixation des anticorps par action spécifique. L'urée et la thiou-rée favorisent la fixation des anticorps. L'ensemble des résultats obtenus avec cette méthode éclaire d'un jour nouveau le mécanisme de la sensibilisation allergique.

Traitement de la pollinose avec les extraits de pollens par la méthode des quadrillages cutanés MM. Jean Blamoutier et L. Guibert proposent une nouvelle technique de désensibilisation dans le traitement du rhume des foins. Ils pratiquent un quadrillage cutané très superficiel fait d'une vingtaine de traits verticaux et horizontaux sur les-quels ils étalent quelques gouttes d'extrait, le plus souvent de pollens, de graminées dont la dilution, pouvant varier de 1/1000 000 à 1 pour 100 , sera choisie suivant $\Gamma$ intensité de la cutí-réaction faite au préalable avec l'extrait sec. La reaction cherchée doit être ædémateuse; son intensité diminuera, lors des quadrillages suivants, pour n'être plus qu'érythémateuse, quand le malade sera protege.

II s'agit là d'un traitement cosaisonnier et non plus présaisonnier. II ne sera commence qu'au debut du rhume des foins et sera renouvelé une ou plusieurs fois, suivant Гimportance et la durée de l'amélioration constatée: quatre quadrillages en moyenne suffisent pour la saison.

Les auteurs ont applique cette méthode, en 1959, à 65 malades, avec d'excellents résultats d'ensemble.

Proceedings - Gesellschaftsberichte - Sociétés

235

Syndrome de Ménière d'origme allergique

MM. Wolfromm, Vialatte, Clerg, Lagrue et Mme Lagrue rapportent Tobservation d'un homme de 46 ans, dont la maladie s'aggravait progressivement depuis 6 ans, aboutissant à une infirmité importante avec vertiges, bourdonnements d'oreilles et surdité. L'origine allergique put être 
démontrée sur les arguments suivants: tests cutanés positifs aux pneumallergènes (poussières, plumes, poils de chat); remission de la maladie à la suite du traitement, avec récupétation de Taudition; rechute à Tarrêt du traitement et nouvelle remission à sa reprise; surtout aggravation des symptômes à plusieurs reprises, à la suite d'injections de doses trop élevées d'allergènes. Urticaire, cystalgies et bactériurie - Tentative de désensibilisation spécifique à l'aide des germes urinaires

MM. Edwin Sidi, A. Reinberg, Mme A. Gervais rapportent le cas d'une femmede 28 ans, atteinte d'une urticaire rebelle, généralisée, évoluant depuis 7 ans, souffranten outre de cystalgies, avec aggravation de 1'urticaire lors de la delta-cortisono-thérapie, et disparition de celle-ci et de la cystalgie par le Furadoïne. Test fortement positif auxgermes urinaires (staphylocoques pathogènes) de la malade, non à d'autres germes età d'autres substances utilisées. La désensibilisation spécifique conduisit à une amelioration remarquable. Pierre Blamoutier 4. Tagung der Gesellschaft für Allergie- und Asthma-forschung in der DDR vom 19.-21. Juni 1959 in Weimar Hauptthemen der Tagung waren Arzneimittelallergie und Infektallergie. Die wissenschaftliche Leitung lag in den Händen von Prof. Dr. A. Sylla, Cottbus, und Dr. D. G. R. Findeisen, Charité, Berlin.

Zum 1. Thema hielt Hansen (Heidelberg) das einleitende Referat. Er betonte, daß die Zahl der Medikamente, wie auch der möglichen allergischen Erscheinungen groß ist und letztere von Jahr zu Jahr zunehmen, sei es durch die Erweitcrung des Arznei-mittelschatzes oder auch durch die zunehmende «Lässigkeit» von Laien wie Ärzten bei der Verwendung der Medikamente. Ebenso vielfältig ist auch die Erscheinungsform medikamentös-allergischer Reaktionen, ohne daß bisher gesagt werden könnte, wodurch die Organwahl im Einzelfall erfolgt. Mehr oder weniger ausgeprägte Schockbilder oder kutane Veränderungen werden relativ häufig diagnostiziert. Schwieriger ist die Diagnose-stellung bei viszeralen Reaktionen, zumal bei chronisch verlaufenden Erkrankungen, z. B. bei Periarteriitis nodosa, beim Erythematodes. Aus der Vielzahl der Medikamente werden nur einzelne besonders wichtige herausgegriffen und deren allergische Neben-wirkungen besprochen, so die Serumallergie als Prototyp allergischer Reaktionen, allergiebedingte Zwischenfälle nach Vakzinationen, Insulin, Hydantoinderivaten, Anti-histaminica, Pyramídon und Antibiotica. Neben dem Schock ist die Agranulozytose die gefürchtetste allergische Komplikation. Oft kann die allergische Genese von Erkrankungen durch genaue Anamnese oder ex juvantíbus (Karenz) geklärt werden. Für Klinik und Forschung stehen auch komplizierte serologische Verfahren zur Ver-fügung.

S. Stüttgen (Düsseidorf) wies auf die Vielfalt morphologischer und funktioneller allergischer Veränderungen an der Haut hin die im wesentlichen durch die Haut-schicht bestimmt werden in der die Antigen-Antikörper-Reaktion abläuft. Schwierig ist die Abgrenzung zwischen allergischer und toxischer Genese im Einzelfall sogar oft 